\title{
Distinct zeros of functions in the Selberg class
}

\author{
by \\ K. SRINIVAS (Chennai)
}

1. Introduction. Selberg $[S]$ defined a class of Dirichlet series that admit analytic continuation, functional equation and an Euler product. The prototypical example of Selberg's class is the classical Riemann zeta function. In the same paper, Selberg formulated several fundamental conjectures. These conjectures have spectacular consequences (see for example the paper by Murty $[\mathrm{M}]$ and Kaczorowski and Perelli $[\mathrm{KP}])$. In this paper we study a question suggested by the properties of the Selberg class.

More precisely, the Selberg class $\mathcal{S}$ (see $[\mathrm{S}]$ ) is defined by the following axioms:

(i) (Dirichlet series) Every $F \in \mathcal{S}$ is a Dirichlet series

$$
F(s)=\sum_{n=1}^{\infty} a(n) n^{-s}
$$

(with $s=\sigma+i t$ ) absolutely convergent for $\sigma>1$.

(ii) (Analytic continuation) There exists an integer $m \geq 0$ such that $(s-1)^{m} F(s)$ is an entire function of finite order.

(iii) (Functional equation) $F \in \mathcal{S}$ satisfies a functional equation of the type $\varphi(s)=w \overline{\varphi(1-\bar{s})}$ where

$$
\varphi(s)=Q^{s} \prod_{i=1}^{d} \Gamma\left(\lambda_{i} s+\mu_{i}\right) F(s) ;
$$

here $w$ is a complex number with absolute value $1, Q(>0), \lambda_{i}(>0), \Re \mu_{i}$ $(\geq 0)$ are certain constants.

(iv) (Ramanujan Hypothesis) For every $\varepsilon>0, a(n)=O\left(n^{\varepsilon}\right)$.

(v) (Euler product) $F \in \mathcal{S}$ satisfies $\log F(s)=\sum_{n=1}^{\infty} b(n) n^{-s}$, where $b(n)=0$, unless $n=p^{m}$ with $m \geq 1$, and $b(n)=O\left(n^{\theta}\right)$ for some $\theta<1 / 2$.

For $F \in \mathcal{S}$ define $\operatorname{deg} F=2 \sum_{i=1}^{d} \lambda_{i}$ to be the degree of $F$.

2000 Mathematics Subject Classification: Primary 11M41; Secondary 11M26. 
Let $F, G \in \mathcal{S}$. Define the counting function $D(T ; F, G)$ of the distinct non-trivial zeros, counted with multiplicity, of the functions $F$ and $G$ by

$$
D(T ; F, G)=\sum_{\substack{0 \leq \Re \varrho \leq 1 \\ 0 \leq \Im \varrho \leq T}} \max \left(m_{F}(\varrho)-m_{G}(\varrho), 0\right),
$$

where $\varrho$ runs over the zeros of $F(s) G(s)$ and is counted without multiplicity and $m_{F}(\varrho), m_{G}(\varrho)$ denote the multiplicities of zeros of $F$ and $G$ respectively. Also define

$$
D(T)=D(T ; F, G)+D(T ; G, F)=\sum_{\substack{0 \leq \Re \varrho \leq 1 \\ 0 \leq \Im \varrho \leq T}}\left|m_{F}(\varrho)-m_{G}(\varrho)\right|,
$$

with the same convention about $\varrho$.

In $[\mathrm{MM}]$, Ram Murty and Kumar Murty proved that if $F \neq G$, then $D(T)=\Omega(T)$.

Assuming that $F$ and $G$ are orthogonal in the sense of Selberg (see [S]) and a certain density hypothesis, Bombieri and Perelli (see [BP]) proved that both $D(T ; F, G)$ and $D(T ; G, F)$ are $\gg T \log T$, provided $\operatorname{deg} F=\operatorname{deg} G$.

The reader may refer to the excellent survey articles by Kaczorowski and Perelli $[\mathrm{KP}]$ and Ram Murty $[\mathrm{M}]$ for general information on the Selberg class and also for a discussion on the distinct zeros and independence results for zeros of functions in the Selberg class.

In this note we propose to study the gaps between the zeros counted by the function $D(T ; F, G)$.

More precisely we prove the following

TheOREM. Let $F$ and $G$ be two distinct functions in $\mathcal{S}$. Assume that $\operatorname{deg} F \geq \operatorname{deg} G$. Then for every sufficiently large $T$, there exists a zero $\varrho=$ $\beta+i \gamma$ of $F G$ with $m_{F}(\varrho)>m_{G}(\varrho)$ such that $|T-\gamma| \leq C_{1} \log \log T$, where $C_{1}$ is a large positive constant.

We obtain the following

COROLlary 1. Under the hypotheses of the Theorem, let $\gamma_{1} \leq \gamma_{2} \leq \ldots$ denote the ordinates of the zeros of $F G$ with $m_{F}\left(\varrho_{i}\right)>m_{G}\left(\varrho_{i}\right), i=1,2, \ldots$ Then

$$
\gamma_{n+1}-\gamma_{n}=O\left(\log \log \left(\left|\gamma_{n}\right|+100\right)\right) .
$$

COROLlary 2. Under the hypotheses of the Theorem,

$$
D(T ; F, G) \geq C_{2} \frac{T}{\log \log T},
$$

where $C_{2}>0$ is a constant.

REMARKs. (1) The global number of distinct zeros given completely unconditionally by Murty and Murty $([\mathrm{MM}])$ is larger than ours. However, 
their method only deals with the symmetric difference of zeros reflected by the counting function $D(T)$, whereas the present paper deals with the asymmetric difference between the zeros reflected by $D(T ; F, G)$, which is a difficult problem and therefore seems interesting.

(2) The main idea in this paper has its origin in the papers [RS] and $[\mathrm{BRSS}]_{1}$, where it has been shown that under suitable conditions the quotient of finite products of translates of the Riemann zeta function has infinitely many poles. Moreover, the gaps between the poles have also been obtained (see also $[\mathrm{BRSS}]_{2},[\mathrm{BRSS}]_{3}$, for various results in this direction).

Acknowledgements. The author thanks Professor Alberto Perelli for proposing the problem, suggesting the relevant literature and encouragement. He also thanks Professors R. Balasubramanian, M. Ram Murty and K. Ramachandra for their support.

2. Notation. Throughout the paper the capital letters $C_{1}, C_{2}, \ldots$ will denote positive constants. We write $f(x) \ll g(x)$ or $f(x)=O(g(x))$ to mean $|f(x)|<C_{3} g(x)$. All the implied constants arising from $\ll$ and $O$ are effective. We fix $H=D \log \log T$ (except in Lemma 1), where $D$ is a large positive constant.

3. Basic lemmas. We need the following lemmas for the proof of the Theorem.

Lemma 1. Let $A>0$ be an integer constant, $B=A+2, a_{1}, a_{2}, \ldots$ be complex numbers with $a_{1}=1,\left|a_{n}\right| \leq n^{A} H^{r \varepsilon / 8}$, where $0<\varepsilon \leq 1 / 2$, $r \geq\left[(200 A+200) \varepsilon^{-1}\right]$, let $\lambda_{1}, \lambda_{2}, \ldots$ be real numbers with $\lambda_{1}=1$ and $1 / C \leq \lambda_{n+1}-\lambda_{n} \leq C$ where $C \geq 1$ is a constant. Then $f(s)=\sum_{n=1}^{\infty} a_{n} / \lambda_{n}^{s}$ is analytic in $\sigma \geq A+2$. Let $K \geq 30, U_{1}=H^{1-\varepsilon / 2}$. Assume that $K_{1}=$ $(H C)^{12 A} K, H \geq\left(120(A+2)^{2} C^{2 A+4}\left(4 r C^{2}\right)^{r}\right)^{100 / \varepsilon}+(100 r(A+2))^{20} \log \log K_{1}$, and that there exist $T_{1}, T_{2}$ with $0 \leq T_{1} \leq U_{1}, H-U_{1} \leq T_{2} \leq H$ such that uniformly in $\sigma \geq 0$ we have

$$
\left|f\left(\sigma+i T_{1}\right)\right|+\left|f\left(\sigma+i T_{2}\right)\right| \leq K,
$$

where $f(s)$ is assumed to be analytically continuable in $(\sigma \geq 0,0 \leq t \leq H)$. Then

$$
\frac{1}{H} \int_{0}^{H}|f(i t)|^{2} d t \geq\left(1-10 r C^{2} H^{-\varepsilon / 4}-100 B H^{-1} \log \log K_{1}\right) \sum_{n \leq H^{1-\varepsilon}}\left|a_{n}\right|^{2} .
$$

Proof. This result is due to R. Balasubramanian and K. Ramachandra (see [R, p. 45]).

Lemma 2. Suppose the Dirichlet series $f(s)=\sum_{n=1}^{\infty} a_{n} / n^{s}$ with $a_{n}=$ $O\left(n^{\varepsilon}\right)$ admits an analytic continuation in an infinite system of rectangles 
$R(T-H, T+H)$ defined by $\{\sigma \geq 0, T-H \leq t \leq T+H\}$ and that $|f(s)|=$ $O\left(\exp \left(C_{4}(\log T)^{2}\right)\right)$ there. Then for all $H \geq C_{5} \log \log T$, we have

$$
\frac{1}{H} \int_{T-H}^{T+H}|f(i t)|^{2} d t \geq C_{6} \sum_{n \leq H^{1 / 2}}\left|a_{n}\right|^{2} .
$$

Proof. The proof follows from Lemma 1 on taking $\lambda_{n}=n, C=1$ and fixing $\varepsilon=1 / 2$ in Lemma 1 .

Lemma 3. Let $F \in \mathcal{S}$. If $\varrho=\beta+i \gamma$ runs through the zeros of $F(s)$, then

$$
\frac{F^{\prime}(s)}{F(s)}=\sum_{|t-\gamma| \leq 1} \frac{1}{s-\varrho}+O(\log |t|)
$$

uniformly for $-2 \leq \sigma \leq 2,|t| \geq 30$.

Proof. This result is well known for the Riemann zeta-function. The proof for the functions in $\mathcal{S}$ is exactly the same since the Riemann-von Mangoldt formula holds for Selberg class (see [T, p. 217]).

Lemma 4. Let $F \in \mathcal{S}$. In any subinterval of length 1 in $[T-H, T+H]$ there are lines $t=t_{0}$ such that

$$
\left|F\left(\sigma+i t_{0}\right)\right|^{-1}=O\left(\exp \left(C_{7}(\log T)^{2}\right)\right)
$$

uniformly in $\sigma \geq-2$.

Proof. Let $I_{0}$ denote a typical rectangle of unit height in $-2 \leq \sigma \leq 2$, $T-H \leq t \leq T+H$. Let $\varrho=\beta+i \gamma$ denote a zero of $F(s)$ in $I_{0}$. Then the number of such zeros, by the Riemann-von Mangoldt formula, is less than $C_{8} \log T$. Therefore, if we break the rectangle $I_{0}$ into equal subrectangles of height $1 /\left(20 C_{8} \log T\right)$, then by the pigeon-hole principle, there is at least one subrectangle $I_{1}$ of $I_{0}$ where $F(s)$ is zero-free. Let $t=t_{0}$ denote the middle horizontal line in $I_{1}$. Clearly $\left|t_{0}-\gamma\right|>1 /\left(40 C_{8} \log T\right)$. Therefore, $\sum_{\left|t_{0}-\gamma\right| \leq 1} 1 /|s-\varrho|<C_{9}(\log T)^{2}$. Now from Lemma 3 , it follows that

$$
\left|\frac{F^{\prime}\left(\sigma+i t_{0}\right)}{F\left(\sigma+i t_{0}\right)}\right|=O\left((\log T)^{2}\right)
$$

uniformly for $\sigma \geq-2$.

To complete the proof we observe that for $\sigma \geq-2$, we have

$$
\log F\left(\sigma+i t_{0}\right)-\log F\left(2+i t_{0}\right)=\int_{\sigma}^{2} \frac{F^{\prime}\left(r+i t_{0}\right)}{F\left(r+i t_{0}\right)} d r=O\left((\log T)^{2}\right)
$$

and

$$
|\log | F\left(\sigma+i t_{0}\right)||=\left|\Re \log F\left(\sigma+i t_{0}\right)\right| \leq\left|\log F\left(\sigma+i t_{0}\right)\right| .
$$


Therefore

$$
\log \left|\frac{1}{F\left(\sigma+i t_{0}\right)}\right|=O\left((\log T)^{2}\right) .
$$

Hence the lemma follows.

Lemma 5. Let $z=x+i y$ be a complex variable with $|x| \leq 1 / 4$. Then

$$
\left|\exp \left((\sin z)^{2}\right)\right| \leq 2 \quad \text { for all } y
$$

and if $|y| \geq 2$, then

$$
\left|\exp \left((\sin z)^{2}\right)\right| \leq 2(\exp \exp |y|)^{-1} .
$$

Proof. This is a double order decaying kernel developed and extensively used by Ramachandra (see Lemma 2.1 of $[R]$ for details).

Lemma 6. Let $F$ and $G$ be two distinct functions in $\mathcal{S}$ with $\operatorname{deg} F \geq$ $\operatorname{deg} G$. Let

$$
f(s)=\frac{G(s-1)}{F(s-1)}=\sum_{n=1}^{\infty} \frac{n c_{n}}{n^{s}},
$$

where $c_{n}$ 's are the coefficients of the Dirichlet series for $G / F$. Assume that $f$ has no poles in the horizontal strip $T-H \leq t \leq T+H$. Then

$$
f(s)=O(1)
$$

uniformly in $R(T-H, T+H)$ provided $H \geq C_{10} \log \log T$.

Proof. Clearly $f(s)=O(1)$ for $\sigma>2$. From the functional equation for $F$ and $G$, we obtain

$$
f(s)=\frac{W_{G}}{W_{F}}\left(\frac{Q_{G}}{Q_{F}}\right)^{3-2 \sigma} T^{(3 / 2-\sigma)(\operatorname{deg} G-\operatorname{deg} F)} \frac{G(2-s)}{F(2-s)} .
$$

Therefore,

$$
f(s)=O\left(\left(\frac{Q_{G}}{Q_{F}}\right)^{3-2 \sigma}\right)+O(1)
$$

uniformly in $\sigma<1$, provided $\operatorname{deg} F \geq \operatorname{deg} G$.

Now, assume that $f(s)$ has no poles in the region $1 \leq \sigma \leq 2, T-H \leq$ $t \leq T+H$. Let $s_{1}=\sigma_{1}+i t_{1}$ be fixed, where $1 / 2 \leq \sigma_{1} \leq 5 / 2, T-H / 200 \leq$ $t_{1} \leq T+H / 200$. Consider the function

$$
D(s)=f(s) \exp \left(\sin \left(\frac{s-s_{1}}{10}\right)^{2}\right) .
$$

We shall apply the maximum modulus principle to the function $G(s)$ in the rectangle bounded by the lines $\sigma=1 / 2, \sigma=5 / 2, t=T-H / 20$ and $t=T+H / 20$. 
Note that $f(s)=O(1)$ on the left vertical line as well as right vertical line, and on the horizontal lines,

$$
G(s-1)=O\left(T^{C_{11}}\right)
$$

and by Lemma 4 we can assume without loss of generality that

$$
\frac{1}{F(s-1)}=O\left(\exp \left(C_{12}(\log T)^{2}\right)\right) .
$$

Therefore, we obtain

$$
f(s)=O\left(T^{C_{11}} \exp \left(C_{12}(\log T)^{2}\right)\right) .
$$

We observe that since

$$
\left|\Re \frac{s-s_{1}}{10}\right| \leq \frac{1}{4} \quad \text { and } \quad\left|\Im \frac{s-s_{1}}{10}\right| \geq C_{13} \log \log T,
$$

by Lemma 5 , on the horizontal lines the factor multiplying $f(s)$ makes the product very small. This is the reason why we had to choose $H$ to be greater than a large positive constant times $\log \log T$.

Therefore, by the maximum modulus principle, it follows that

$$
\left|D\left(s_{1}\right)\right|=\left|f\left(s_{1}\right)\right|=O(1) .
$$

Since $s_{1}$ was arbitrary, the lemma follows.

Lemma 7. Let $f$ be as defined in Lemma 6 . Then $f$ cannot be absolutely convergent in $\sigma>3 / 2-\varepsilon$ for any $\varepsilon>0$. Moreover,

$$
\sum_{n \leq X}\left|c_{n}\right|^{2} n^{2} \rightarrow \infty \quad \text { as } X \rightarrow \infty .
$$

Proof. Let $a_{n}(F)$ and $a_{n}(G)$ denote the $n$th coefficients of the Dirichlet series for $F$ and $G$ respectively. It is well known that if $a_{p}(F)=a_{p}(G)$ and $a_{p^{2}}(F)=a_{p^{2}}(G)$ for all but finitely many primes $p$, then $F=G$ (see [MM]). Using the functional equation for $F$ and $G$, it can be easily shown that if $F \neq G$, then the function $G / F$ cannot converge absolutely in $\sigma \geq 1 / 2-\varepsilon$ for any $\varepsilon>0$. This establishes the first part of the lemma.

In particular,

$$
\sum_{n \leq X} \frac{\left|c_{n}\right|}{n^{1 / 4}} \rightarrow \infty \quad \text { as } X \rightarrow \infty .
$$

By the Cauchy-Schwarz inequality, we obtain

$$
\sum_{n \leq X} \frac{\left|c_{n}\right|}{n^{1 / 4}} \leq\left\{\sum_{n \leq X}\left|c_{n}\right|^{2} n^{2}\right\}^{1 / 2}\left\{\sum_{n \leq X} \frac{1}{n^{5 / 2}}\right\}^{1 / 2} .
$$

As the second factor on the right hand side of the above expression is bounded, the lemma follows. 
4. Conclusion of the proof. By Lemma 6 one can apply Lemma 2 with $H=D \log \log T$ and obtain the inequality

$$
1 \gg \frac{1}{H} \int_{T-H}^{T+H}|f(i t)|^{2} d t \gg \sum_{n \leq H^{1 / 2}} n^{2}\left|c_{n}\right|^{2},
$$

but this leads to a contradiction by Lemma 7 on taking $H \geq X$.

The proofs of the Corollaries 1 and 2 are immediate.

\section{References}

$[\text { BRSS }]_{1}$ R. Balasubramanian, K. Ramachandra, A. Sankaranarayanan and K. Srinivas, Notes on the Riemann zeta-function, III, Hardy-Ramanujan J. 22 (1999), 23-33.

[BRSS $]_{2}-,-,-,-$, Notes on the Riemann zeta-function, IV, ibid., 34-41.

[BRSS $]_{3}-,-,-,-$, Notes on the Riemann zeta-function, $V$, ibid. 23 (2000), 2-9.

[BP] E. Bombieri and A. Perelli, Distinct zeros of L-functions, Acta Arith. 83 (1998), 271-281.

[KP] J. Kaczorowski and A. Perelli, The Selberg class: a survey, in: Number Theory in Progress, Proc. Conf. in Honor of A. Schinzel, K. Győry et al. (eds.), de Gruyter, Berlin, 1999, 953-992.

[M] M. R. Murty, Selberg conjectures and Artin L-functions, Bull. Amer. Math. Soc. 31 (1) (1994), 1-14.

[MM] M. R. Murty and V. K. Murty, Strong multiplicity one for Selberg's class, C. R. Acad. Sci. Paris 319 (1994), 315-320.

[R] K. Ramachandra, On the Mean-Value and Omega-Theorems for the Riemann Zeta-Function, Tata Inst. Fund. Res. Lectures on Math. and Phys. 85, Springer, Berlin, 1995.

[RS] K. Ramachandra and A. Sankaranarayanan, Notes on the Riemann zeta-function, II, Acta Arith. 91 (1999), 351-365.

[S] A. Selberg, Old and new conjectures and results about a class of Dirichlet series, in: Collected Papers, 2, Springer, 1991, 47-63.

[T] E. C. Titchmarsh, Theory of the Riemann Zeta-Function, 2nd ed., Clarendon Press, Oxford, 1986.

The Institute of Mathematical Sciences

Tharamani P.O.

Chennai 600 113, India

E-mail: srini@imsc.ernet.in

Received on 25.9.2000

and in revised form on 10.9.2001 